\title{
Application of flow cytometry in diagnosing lymphomas in dogs and cats
}

\author{
OLGA ANIOEEK, ZDZISŁAW GAJEWSKI, SŁAWOMIR GIZINSSKI
}

Faculty of Veterinary Medicine, Department of Large Animal Diseases with Clinic, Warsaw University of Life Sciences, Warsaw, Poland

\begin{abstract}
Classification of types of lymphomas is done by interpreting cell morphology results obtained in cytological and/or histological examinations. In recent years, additional methods like immunocytochemistry (ICC), immunohistochemistry (IHC), immunophenotyping by flow cytometry and polymerase chain reaction (PCR) for antigen receptor rearrangements (PARR), have been used to diagnose and classify lymphomas. Unfortunately, none of these methods is completely specific and sensitive. Thus, a combination of several diagnostic methods or use of all available techniques allows for evaluation of morphological properties of cells like their maturity and diversification. Owing to the use of sets of antibodies it is possible to identify the phenotype of hyperplastic cells as well as their origin. Combination of results obtained through phenotypical analysis with flow cytometry examination with morphological, histological and genetic testing enables a detailed analysis of, in this case, lymphoproliferative diseases including reaction changes, primary and secondary immunological deficits as well as autoimmune diseases.
\end{abstract}

Key words: lymphoma, flow cytometry, immunophenotyping.

(Centr Eur J Immunol 2014; 39 (3): 327-330)

Lymphomas are the most common type of lymphoproliferative malignant neoplasms in dogs and cats. They comprise a diverse group as far as morphology, phenotype, clinical manifestations, signs, prognosis and treatment are concerned [1]. The majority of lymphomas in dogs (80-85\% of cases) affect nodes with B-cell lymphomas being, just like in humans, the most common variant of the disease [2]. Nevertheless, T-cell type lymphomas are also diagnosed [3]. Apart from lymphomas originating from B-cells and T-cells, a certain percentage of tumours not displaying the expression of any of the markers defining their connection to the line of T- or B-lymphocytes (null, non-B, non- $\mathrm{T}$ ) or possessing markers characteristic of both types of lymphocytes can also be observed. Prognosis depends not only on the cell line of the neoplasm, but also on the location, cell characteristics and the tumour development stage. In cats, the diagnosis is more difficult due to the fact that extranodal, digestive and respiratory system lymphomas are observed [4]. Digestive tract lymphoma is quite frequent, however it is difficult to diagnose as taking of the biological sample is difficult. Moreover, plasmocyte infiltration and reactive hyperplasms present during inflammatory processes further hinder cytological and histopathological examinations. Additionally, lymphomas with nonhomogeneous groups of lymphocytes containing reactive plasma cells, lymphocytes and other inflammatory cells pose yet another problem.

\section{Immunophenotyping by flow cytometry}

One of the aims of immunophenotyping of flow cytometry is to determine the type of lymphocytes in samples submerged in liquid according to light scattering capabilities and expression of surface markers. This method allows for quick quantitative and qualitative evaluation of population of hematopoietic cells, etc. In this case, it is used in order to discern lymphocytes affected by the neoplasm [5, 6]. Lymphocytes have a large number of various particles, which allow for their identification. Some of them, known as markers of main cell lines, are present in all lymph cells e.g. CD45, other subpopulations of T lymphocytes (CD2, CD3, CD5, CD7) and $\mathrm{B}$ (CD19, CD20, CD22, CD79). In certain situations some markers of a single cell line may be subject to co-expression on cells from a different line e.g. from CD5, which is often present is some B cell population. Furthermore, a significant number of markers are present only temporarily, in a determined sequence on certain cells, during maturation, differentiation and activation. Markers of maturation for one line may be markers of activation for other lines [7].

Correspondence: Olga Aniołek, Faculty of Veterinary Medicine, Department of Large Animal Diseases with Clinic, Warsaw University of Life Sciences, Nowoursynowska 100,02-797 Warsaw, Poland, e-mail: olgaaniolek@op.pl 


\section{Antibody panels used in diagnosis of lymphomas in dogs and cats}

Nowadays, when attempting to detect a lymphoma, it is recommended to determine the phenotype using the biggest range of available antibodies. Use of flow cytometry for diagnosing a lymphoma is limited by the fact that neoplastic lymphocytes may not possess the same phenotype as their non-neoplastic equivalents. Moreover, they may exhibit surface markers inappropriate to their level of differentiation or exhibit additional markers not connected to neoplastic cells. Antibody panels evaluating lymphoid tissue with a smaller number of antibodies evaluating the granulocyte line, monocytes, erythroid line and megakaryocyte cells are available for cats and dogs (Table 1). Owing to that fact, it is strongly recommended to use an antibody panel that characterises the population of neoplastic cells (Table 2). Some phenotypes and expression models possess specific prognostic markers (Table 3) [812]. Leukaemia or lymphoma induced lymphocytosis is characterised by homogenic expansion of cells with an identical phenotype. Reactive lymphocytes can be heterogenic and consist of a few subgroups of lymphocytes. An important exception to this conception may be the presence of homogenic monoclonal expansion of CD8 T cells observed in Ehrlichia canis [13].

Examination of lymphoid markers on hyperplastic cells provides a lot of valuable information facilitating differen- tial diagnosis. It also enables identification of types of lymphomas, which are very similar in the cytological or histological picture. Small cell lymphocytic lymphoma/chronic leukaemia exhibits morphological similarity (especially in cytological examination) to lymphoplasmacytic lymphoma and mantle cell lymphoma. All these types of hyperplasms may exhibit blood smear during the course of the disease and have a similar clinical picture. In differential diagnosis of these hyperplasms, an appropriate panel of monoclonal antibodies (CD5, CD20, CD22, CD23) and detection of Ig in cytoplasm or on the surface of neoplastic cells is highly useful. Differentiation between lymphoma and nonhyperplastic conditions in lymph nodes is a very important and difficult task. Attempts to differentiate between lymphoma of follicle centre (follicular lymphoma - FL) and reactive hyperplasms in lymph nodes may serve as a good example. In this case, identification of the monoclonal protein, i.e. $\kappa$ or $\lambda$ immunoglobulin chains is an indicator of the hyperplastic character of cells.

\section{Taking samples for flow cytometric analysis}

Blood sample and body cavity fluids (cerebrospinal fluid, peritoneal fluid, exudative fluid, transudative fluid, etc.) for immunophenotyping by flow cytometry are to be placed in a test tube with EDTA. Samples obtained

Table 1. Surface markers of leukocytes in dogs and cats detected using flow cytometry

\begin{tabular}{|c|c|c|}
\hline Marker & Base cell type & Comment \\
\hline CD1 & Dendritic cell & Subpopulation of B-cells and monocytes may also indicate CD1 \\
\hline CD3 & T cells & \\
\hline CD4 & T helper cell & $\begin{array}{l}\text { Dogs' neutrophil granulocytes may express CD3; also monocytes, } \\
\text { macrophages and dendritic cells }\end{array}$ \\
\hline \multicolumn{3}{|c|}{ CD4/CD8 double positive } \\
\hline CD5 & $\mathrm{T}$ cells & In some species, CD5 may also be present in B cells \\
\hline CD8 & Cytotoxic T cell & Also in the NK subgroup \\
\hline CD14 & Monocytes & Also in some types of macrophages \\
\hline CD18 & All leukocytes & Greater intensity on granulocytes and monocytes \\
\hline $\mathrm{CD} 21, \mathrm{CD} 22$ & Mature B cells & Absent on plasma cells \\
\hline CD34 & Hematopoietic stem cell & \\
\hline CD45 & $\begin{array}{l}\text { All hematopoietic stem cells (with the } \\
\text { exception of erythroid cells) }\end{array}$ & $\begin{array}{l}\text { CD } 45 \text { may be absent or with a lower expression on neoplasmic } \\
\text { cells }\end{array}$ \\
\hline CD79a or CD79b & B cell all stages & Intracellular antigen \\
\hline MHC II & $\begin{array}{l}\text { Cell presenting antigen, majority of dog } \\
\text { and cat lymphocytes }\end{array}$ & \\
\hline Surface IgM & Immature B cells & \\
\hline Light chain expression & Antibody producing B cells, plasmacytes & $\begin{array}{l}\text { Expression of } \lambda \text { chain significantly exceeds } \kappa \text { chain in dogs and } \\
\text { cats; present in both benign and malignant tumours }\end{array}$ \\
\hline
\end{tabular}


Table 2. Antibody panel used in flow cytometry

\begin{tabular}{lll}
\hline Type of neoplasm & Morphological characteristics & Common phenotype \\
\hline Dog CLL: T-cell type & Small, medium lymphocytes, often with granularities & CD3+ CD5+ CD8+ \\
\hline Dog CLL: B-cell type & Small lymphocytes & CD21+ CD79+, possible monoclonal gammopathy \\
\hline Cat CLL & Small lymphocytes & CD5+ CD4+ \\
\hline Acute lymphoblastic leukaemia & Large cells & Some are CD45+ CD34+ \\
\hline Diffuse large B-cell lymphoma & Large lymphocytes & CD21+ CD79+ CD1+ CD18+ \\
\hline MALT lymphoma & Small to medium lymphocytes & CD21+ CD79+ CD1-, CD18+ \\
\hline
\end{tabular}

Table 3. Prognostic factors

\begin{tabular}{lll}
\hline Neoplasm type & Changes & Suggested prognosis \\
\hline B-cell type lymphoma & Loss of MHC II & Lower survival rate \\
\hline Dog CLL & B-cell subtype & Lower survival rate in comparison to T-cell CLL \\
\hline Dog CLL & $\begin{array}{l}\text { Atypical subtype (null T- cell, double CD4+/CD8+, } \\
\text { double T/B) }\end{array}$ & \\
\hline ALL or V stage lymphoma & CD34 expression & CD34 ALL expression, rarely V stage lymphoma \\
\hline
\end{tabular}

ALL-acute lymphoblastic leukaemia; CLL-chronic lymphoblastic leukaemia; $M H C$ - major histocompatibility complex

via FNA (fine needle aspiration) from tissues (e.g. lymph nodes) should be placed in test tubes containing Ringer's solution buffered with a small amount of serum (bovine serum is commonly used) in order to stabilize the sample prior to analysis. It is best to contact the diagnostic laboratory in order to establish the procedures concerning transportation of the biological material. Usually, the minimum of $2 \times 10^{6}$ cells is necessary in order to apply a complete panel of immunophenotyping by flow cytometry. It is important that samples from different parts should not come in contact with one another. Material should be cooled down but not frozen. The time of delivery of the biological material to the laboratory is also important as any delay may cause cell death and/or loss of intensity of expression of a given surface marker. Diagnostic material can be preserved for the maximal period of 3 days. Most of the laboratories in America, Europe and Asia use a similar antibody panel for leukocyte characterisation.

Figure 1 shows FNA biopsy of a lymph node.

\section{Summary}

Immunophenotyping by flow cytometry is a method used in research centres but it is becoming available in an increasing number of veterinary laboratories. Flow cytometry is a modern analytic method of biochemical and physical properties of cells. In mere seconds, with unmatched precision and reproducibility, it is possible to detect rare and unusual subpopulations. Morphological properties of a certain hyperplasm are imperturbably important as they give direction to phenotypic tests, but on their own are insufficient. It is important to remember to analyse each

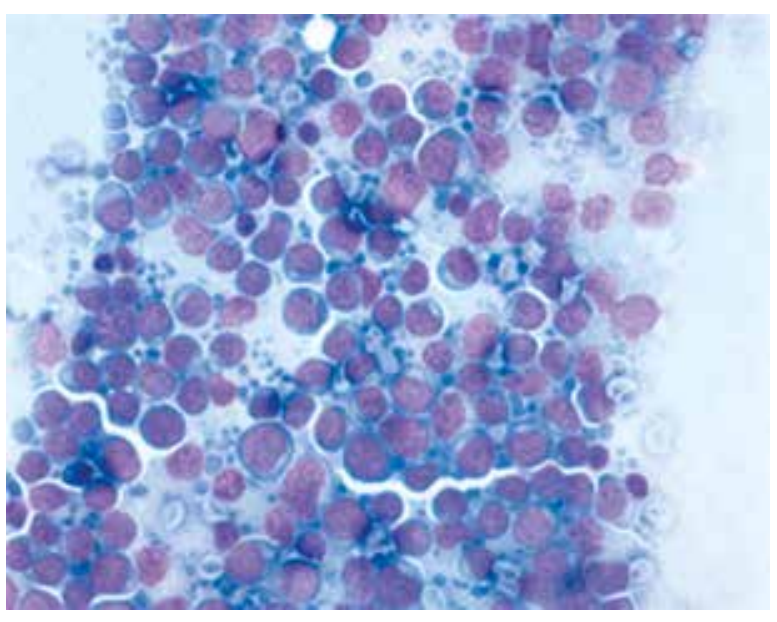

Fig. 1. Cytological picture of fine needle aspiration biopsy of a lymph node. Typical picture of a lymphoma - homogenic population of big lymphoid cells with visible cell nuclei and strongly basophilic cytoplasm. Numerous lymphoglandular bodies, rare small lymphocytes. Magnification $40 \times$, hemacolor stain

and every result individually and correlate it with results of morphological tests carried out on the same material (histopathological, cytogenetic) taking the age of the patient, reactive changes and autoimmune diseases into consideration.

The authors declare no conflict of interest. 


\section{References}

1. Bienzle D, Vernau W (2011): The diagnostic assessment of canine lymphoma: implications for treatment. Clin Lab Med 31: 21-39.

2. Valli VE, San Myint M, Barthel A, et al. (2011): Classification of canine malignant lymphomas according to the World Health Organization criteria. Vet Pathol 48: 198-211.

3. Avery PR, Burton J, Bromberek JL, et al. (2014): Flow cytometric characterization and clinical outcome of CD4+ T-cell lymphoma in dogs: 67 Cases. J Vet Intern Med 28: 538-546.

4. Chino J, Fujino Y, Kobayashi T, et al. (2013): Cytomorphological and immunological classification of feline lymphomas: clinicopathological features of 76 cases. J Vet Med Sci 75: 701-707.

5. Little L, Patel R, Goldschmidt M (2007): Nasal and nasopharyngeal lymphoma in cats: 50 cases (1989-2005). Vet Pathol 44: 885-892.

6. So“zmen M, Tasca S, Carli E, et al. (2005): Use of fine needle aspirates and flow cytometry for the diagnosis, classification, and immunophenotyping of canine lymphomas. J Vet Diagn Invest 17: 323-329.

7. Wilkerson MJ, Dolce K, Koopman T, et al. (2005): Lineage differentiation of canine lymphoma/leukemias and aberrant expression of CD molecules. Vet Immunol Immunopathol 106: 179-196.

8. Williams MJ, Avery AC, Lana SE, et al. (2008): Canine lymphoproliferative disease characterized by lymphocytosis: immunophenotypic markers of prognosis. J Vet Intern Med 22: 596-601.

9. Campbell MW, Hess PR, Williams LE (2012): Chronic lymphocytic leukaemia in the cat: 18 cases (2000-2010). Vet Comp Oncol 11: 256-264.

10. Comazzi S, Gelain ME, Martini V, et al. (2011): Immunophenotype predicts survival time in dogs with chronic lymphocytic leukemia. J Vet Intern Med 25: 100-106.

11. Rao S, Lana S, Eickhoff J, et al. (2011): Class II Major histocompatibility complex expression and cell size independently predict survival in canine B-cell lymphoma. J Vet Intern Med 25: 1097-1105.

12. Burkhard MJ, Bienzle D (2013): Making Sense of Lymphoma Diagnostics in Small Animal Patients. Vet Clin Small Anim 43: 1331-1347.

13. Avery AC, Avery PR (2007): Determining the Significance of Persistent Lymphocytosis. Vet Clin Small Anim 37: 267-282. 